\title{
Final Performance Report to the Department of Energy by Prairie View A\&M University High Energy Physics
}

\author{
Dennis J. Judd \\ Principal Investigator
}

May 14, 1992

DISCLAIMER

\begin{abstract}
This report was prepared as an account of work sponsored by an agency of the United States Government. Neither the United States Government nor any agency thereof, nor any of their employees, makes any warranty, express or implied, or assumes any legal liability or responsibility for the accuracy, completeness, or usefulness of any information, apparatus, product, or process disclosed, or represents that its use would not infringe privately owned rights. Reference herein to any specific commercial product, process, or service by trade name, trademark, manufacturer, or otherwise does not necessarily constitute or imply its endorsement, recommendation, or favoring by the United States Government or any agency thereof. The views and opinions of authors expressed herein do not necessarily state or reflect those of the United States Government or any agency thereof.
\end{abstract}




\section{DISCLAMMIRR}

Portions of this document may be illegible in electronic image products. Images are produced from the best available original document. 


\section{Contents}

I. Project Overview ........................ 1

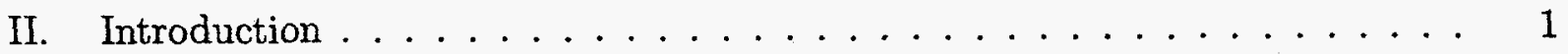

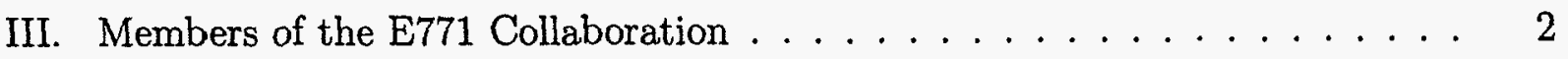

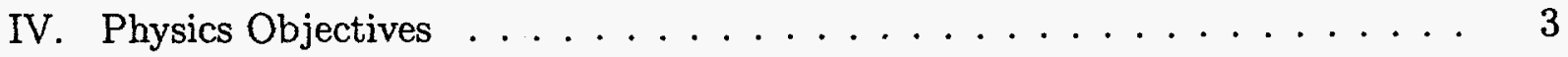

V. Beam Requirements/Experimental Running $\ldots \ldots \ldots \ldots$

VI. Experimental Setup . . . . . . . . . . . . . . . 8

VII. Event Yield/Backgrounds . . . . . . . . . . . . . . 9

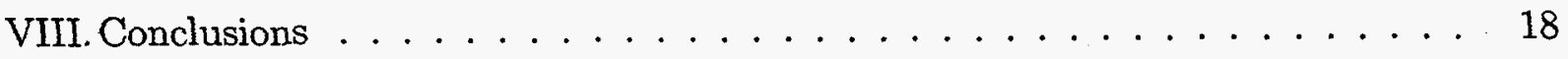

IX. References . . . . . . . . . . . . . . . . . . . 19

X. Prairie View High Energy Physics Publications from 1989-1992 _. . . . . 21

XI. 1990 E705/E771 Status Report . . . . . . . . . . . . . . . 23

XII. 1991 E771 Progress Report. . . . . . . . . . . . . . . . . . . 28

A. Apparatus .......................... 28

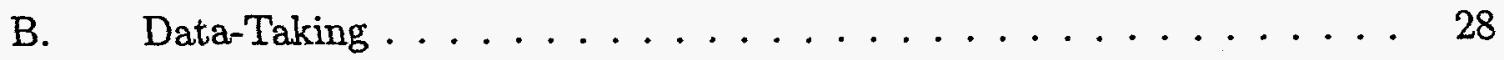

C. Off-line Data Analysis . . . . . . . . . . . . 29

D. Student Support . . . . . . . . . . . . . . 29

XIII. 1992 E771 Progress Report . . . . . . . . . . . . . . . . . . . . 30 
A. The 1991 Run . . . . . . . . . . . . . . . . 30

B. Off-line Data Analysis . . . . . . . . . . . . . . 31 


\section{List of Tables}

I $\quad B$ Meson Decay Table $\ldots \ldots \ldots \ldots \ldots \ldots \ldots \ldots$

II Beam Parameters $\ldots \ldots \ldots \ldots \ldots \ldots \ldots \ldots$

III Calculated $B$ Cross-Sections $\ldots \ldots \ldots \ldots \ldots \ldots$

IV Number of Calculated $\bar{B} B$ events $\ldots \ldots \ldots \ldots \ldots$

V Number of $B \rightarrow J / \psi+\mathrm{X} ; J / \psi \rightarrow \mu^{+} \mu^{-} \ldots \ldots \ldots \ldots$

VI $\chi$ Cross-Sections and Acceptances $\ldots \ldots \ldots \ldots \ldots \ldots \ldots \ldots$ 


\section{Project Overview}

The High Energy Physics (HEP) group at Prairie View A\&M University is a collaborator with Fermi National Accelerator Laboratory (Fermilab), and the universities listed below. The purpose of this collaboration is to contribute to the understanding of heavy quark hadroproduction. Our efforts began in the early 1980's at Fermilab with the study of the charmonium states, $J / \psi$ and $\chi$, (DE-FG-86ER-40297) and presently with the continued studies of the charmonium system and direct photon production (Fermilab experiment E705) and new studies on bottom production (Fermilab experiment E771) in the High Intensity Laboratory (Proton-West Area) of Fermilab. The Prairie View group will, as a part of their task, be directly responsible for a major part of the PWC system upgrade by developing the electronics for the readouts of the PWC pad chambers. Six in all, these chambers, are a part of new multlevel triggering scheme and represents a departure from the triggering methodology of the previous trigger processors in earlier experiments. The Prairie View group is also involved with the Bottom Collider Detector (BCD) Collaboration which is proposing to study bottom production at the Fermilab Collider and at the Superconducting Super Collider (SSC).

\section{Introduction}

The presence of single muons and especially dimuons in final states produced in hadronic interactions has proved to be a valuable indicator that interesting hard physics processes have taken place. These muons provide a mechanism for selecting the relatively rare processes from interactions due-to the total cross-section. In particular, processes involving heavy quarks are flagged cleanly by the presence of muon pairs. We are proposing to use the high rate E705 spectrometer ${ }^{1}$ (shown in Fig. 1) but with a muon trigger system which differs from our earlier ECL-CAMAC processor. ${ }^{2,3}$ The ECL-processor functioned well in Experiments $537^{4}$ and 705) detecting and measuring several heavy quark phenomena which resulted in a final state containing muons. In the current experiment (E771) we operate under a new trigger methodology. The dimuon trigger still looks for two muons as before but** one of the two muons must have a $P_{t}$ of $0.8-1 \mathrm{GeV}$. The mass calculation of E537 and E705 experiments have been abandoned. We will also augment our spectrometer by the addition of a silicon tracker similar to those $e^{5}$ used in other experiments at Fermilab. The present P-West High Intensity Laboratory secondary beam ${ }^{6}$ will need to be upgraded with the addition of sufficient bending power to allow the transport of the $800 \rightarrow 925 \mathrm{GeV} / \mathrm{c}$ primary proton beam to the experiment target.

We desire to continue our studies of hadronicly** produced heavy quark dynamics and share that knowledge within an academic community usually not able to participate in such research. and thereby satisfy to a small extent a growing national need for minority scholars. We have in a single year increased the number of physics majors from one to eleven. 
We have selected students from the special Banneker Honors College and only those with a desire for an advanced degree in physics. By the fall of 1991, we expect at least two graduate students to be enrolled at Texas A\&M University.

Our present grant (DE-AS05-88ER-40457) began June 14, 1988. We have been participating in E705 since its inception in 1981. The current level of funding, $\$ 200,000$ will be matched by $\$ 100,000$ from Prairie View A\&M University plus computing time on university computers. However, upgrades to these computers are required. We will also receive annual $\$ 30,000$ in super computer time on the NEC-SX2 from the Houston Area Research Center, site of the Texas Accelerator Center. In addition the Prairie View A\&M University HEP group is also receiving Department of Energy equipment funds for the construction of electronic equipment to be used on the beauty experiment E771 at Fermilab. The charmonium and direct photon experiment (E705) has completed data taking, accumulating over 7500 magnetic data tapes. We have exceeded the CERN WA70 experimental sample for direct photon production by $\pi^{ \pm}$.

\section{Members of the E771 Collaboration}

University of Athens

Brown University

University of California, Berkeley

University of California at Los Angeles

Duke University

Fermi National Accelerator Laboratory

University of Houston

Indiana University

University of Lecce

Massachusetts Institute of Technology

McGill University

Nanking University 
Northwestern University

University of Pavia

University of Pennsylvania

Prairie View A\&M University

Shandong University

University of South Alabama

University of Virginia

\section{Physics Objectives}

A. The goal of E771 is the measurement of the hadroproduction of bottom in proton-silicon interactions using the $800 \mathrm{GeV} / c$ primary proton beam from the Tevatron. We will look for bottom hadrons and their antiparticles produced via

$$
p \mathrm{Si} \rightarrow \bar{B} B+\mathrm{X}
$$

where either the $B$ or the $\bar{B}$ decays into

$$
J / \psi+\mathrm{X}
$$

with the $J / \psi$ decaying into

$$
J / \psi \rightarrow \mu^{+} \mu^{-}
$$

In addition, we will look for production of bottom hadrons which also decay to single muons.

The cleanest experimental signature for such physics will be events with opposite sign dimuons which reconstruct to the $J / \psi$ mass and which point to a secondary decay vertex located in air as measured by a silicon tracker. The association of the $J / \psi$ with a secondary decay vertex located outside of the target material insures that one is seeing bottom rather than charm production. Since the branching ratio for $B \rightarrow J / \psi+$ anything has been measured by both the ARGUS ${ }^{7}(1.10 \pm 0.19 \%)$ and CLEO ${ }^{8}(1.10 \pm 0.21 \pm 0.23 \%)$ experiments 
and a rough lifetime for bottom is known from the WA75 measurement ${ }^{9}\left(3.0_{-1}^{+2} \times 10^{-13}\right.$ $\mathrm{sec})$, and from the PEP-PETRA measurement ${ }^{10,11}\left(1.2 \pm 0.2 \times 10^{-12} \mathrm{sec}\right)$, it is possible to determine the cross-section for bottom hadroproduction from our observation of isolated $J / \psi$ secondary vertices without the complete reconstruction of the bottom meson final states. The calculation of the cross-section without complete reconstruction of particular final states will depend to a small extent on a model dependent estimate of the efficiency for seeing the secondary vertex. Background to the detection of bottom decay comes primarily from ordinary $J / \psi$ production in which the $J / \psi$ fakes an isolated secondary vertex by being misreconstructed to a point far from the primary vertex because of measurement errors.

Another important result which will be attempted by E771 will be a direct measurement of the lifetime of the bottom mesons and a determination of the size of a lifetime difference (if any) between the charged and neutral $B$. Since most of the center of mass energy of the $B$ is carried away by the massive $J / \psi$ in the decays of interest for E771, and since the silicon tracker can be used to follow the other charged products of the $B$ decay, an accurate determination of the invariant lifetime is possible from the measurement by the silicon of the Lorentz boosted decay length of the $B$ and the measurement of the momentum of the $B$ by the measurement of the momentum of the $J / \psi$ and other associated charged tracks.

B. In addition to the observation of $B \rightarrow J / \psi+$ anything and the determination of the total cross-section and lifetime of bottom meson production, we intend to reconstruct specific exclusive final states of the bottom mesons decaying to $J / \psi$. Complete reconstruction of particular exclusive final states permits a refined measurement of the momentum and lifetime distributions and provides extra constraints for the measurement of the hadroproduction cross-sections and branching ratios for bottom. This complete reconstruction will be possible for some final states. We list in Table I the Cabibbo-favored final states for the bottom meson decays which result in less than or equal to three charged particles. Estimated branching ratios ${ }^{12}$ are provided in some cases and in one particular case $\left(B^{-} \rightarrow J / \psi K^{-}\right)$ an experimental measurement is available. The decay table for the antiparticles is similar of course except for CP violating effects. We note that the $B^{0}$ and $\bar{B}^{0}$ secondary vertices result in an even number of outgoing charged tracks and the $B^{ \pm}$secondary vertices in an odd number of charged tracks.

Most of these relatively simple final states are observable at some level but each has its own special experimental problems. For purposes of the following discussion we will assume no charged $K-\pi$ identification although we are actively pursuing implementing such identification. In the absence of such identification a $K-\pi$ ambiguity will be present for all non-muon charged tracks. This will necessitate two entries in any $J / \psi X^{-} X^{+}$mass plot. The final states involving a $K^{0}$ will require the assignment of that $K^{0}$ to the secondary decay vertex without the presence of a charged track in the silicon tracker and will therefore lead to ambiguities between $K^{0}$ 's produced in the secondary vertex and those from the primary vertex. This will increase the spurious mass combinations. For the case of bottom meson final states containing a $\pi^{0}$, all $\pi^{0} \mathrm{~s}$ in the event are candidates for inclusion in the reconstructed 
Table I: $B$ Meson Decay Table

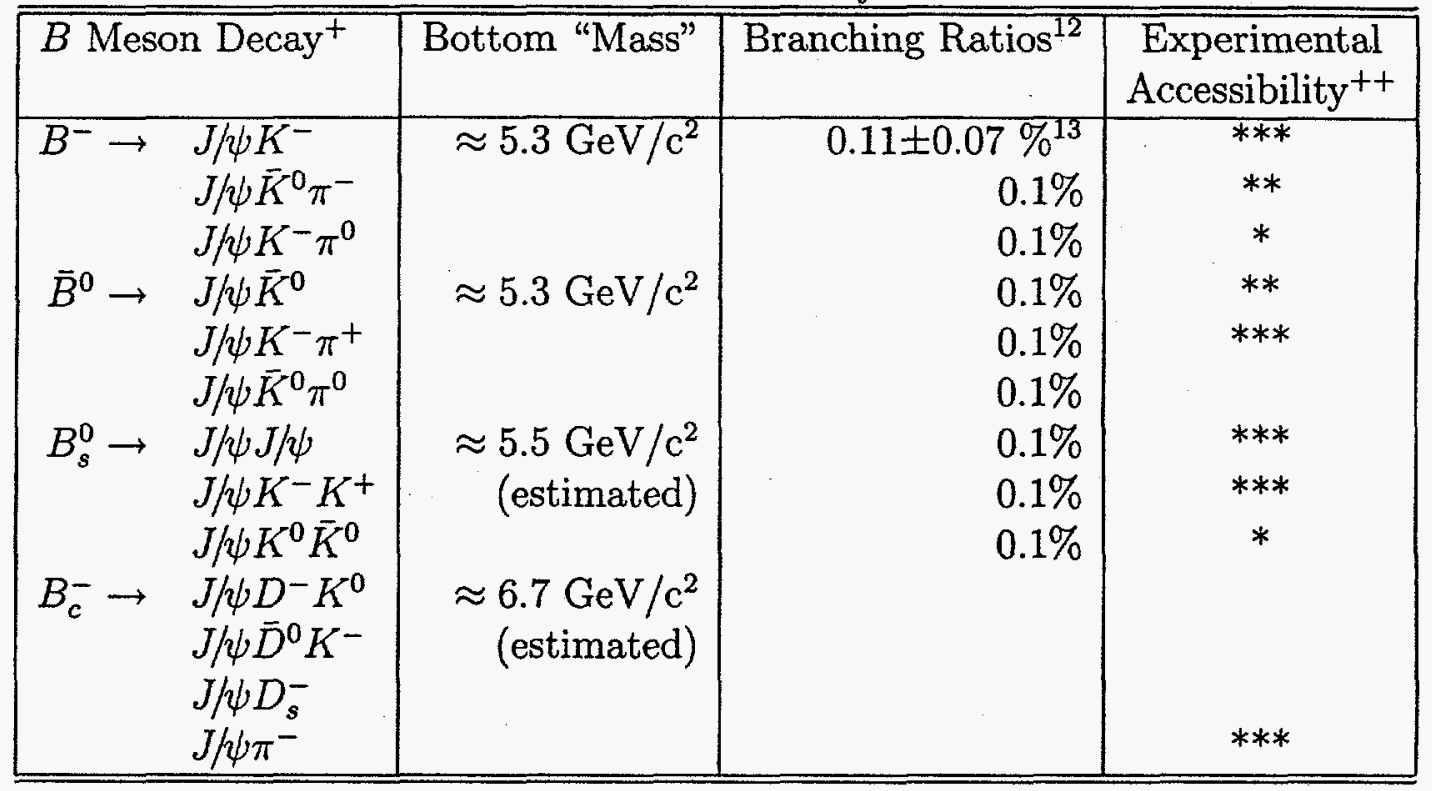

+ The $B_{c}^{-}$can decay directly to $B^{-}, \bar{B}^{0}, \bar{B}_{s}^{0}$ plus anything followed by the subsequent decay of any of the three $B$ mesons into $J / \psi$ plus anything. However, these decays are more complicated to analyze and are not discussed.

++The relative experimental "accessibility" of the final states is roughly indicated by the number of *'s.

secondary vertex. This leads to a large combinatorial background for final states containing a $\pi^{0}$ and a desire to concentrate on only charged final states. Considering all of these factors, we have rendered a qualitative judgement about the experimental accessibility of each final state in Table I. Those final states in which the bottom decays result in only charged particles are particularly suited for reconstruction.

C. The third physics goal of this experiment will be the observation of $\bar{B} B$ mixing by observation of high mass like sign dimuons where each of the muons points to a different secondary vertex. The opposite sign muons from different vertices are due to the semileptonic decays of either charm or bottom and are produced in the process:

$$
p \mathrm{Si} \rightarrow \bar{B}^{0} B^{0}+\mathrm{X}
$$

with the two neutral $B$ mesons each decaying semileptonically.

The same sign dimuons of interest are produced when either the $\bar{B}^{0}$ or the $B^{0}$ have evolved into the conjugate antiparticle and produces the same sign muon as its partner in the semileptonic decay. The backgrounds to same sign dimuons from $\bar{B} B$ mixing can arise from: 
1. The sequential decay of either the $B$ or the $\bar{B}$ into a charmed particle followed by the semileptonic decay of that charmed particle in conjunction with the semileptonic decay of the other $B$ meson. The sign of the lepton produced in the semileptonic decay of the daughter is opposite to the sign of the lepton that would have been produced by the semileptonic decay of the parent, thereby leading to a same sign dimuon background.

2. Direct production of and mixing of $C \bar{C}$ pairs $\left(D^{0}, \bar{D}^{0}\right.$ for example) where the charmed particle evolves into the anti-charmed particle and generates a same sign background to the same sign muons from $B^{0} \bar{B}^{0}$ mixing. However, the mixing of $D^{0}$ and $\bar{D}^{0}$ has been measured to be small, so this is not anticipated to be a large source of background.

3. Production of $\bar{B} B$ pairs with one of the $B$ 's decaying semileptonically and the other decaying nonleptonically but with one of the pions from the nonleptonic decay undergoing $\pi \rightarrow \mu \nu$ decay.

The magnitude of these backgrounds and the techniques for suppressing them are discussed in section VI-C. We will only mention here that these backgrounds can be suppressed since:

1. The same sign dimuon signal from $B \bar{B}$ mixing will have higher mass than the same sign dimuon backgrounds.

2. Secondary vertices can be indentified as bottom decays by their visible mass.

We will investigate the effectiveness of these techniques in eliminating the same sign backgrounds when the mixing experiment is discussed below.

D. A fourth objective of this experiment will be the continuation at higher energies of the measurement of hadronic production of charmonium which we just completed at $300 \mathrm{GeV} / \mathrm{c}$ in E705 ${ }^{7}$. The objective of E705 is to measure $\left(\pi^{ \pm}, p, \bar{p}\right) \mathrm{Li} \rightarrow \chi+$ anything and $\left(\pi^{ \pm}, p, \bar{p}\right) \mathrm{Li} \rightarrow \gamma+$ anything at $300 \mathrm{GeV} / \mathrm{c}$ (DOE Grant No. DE-FG-05-86ER-40297). At the same time as we perform the study of bottom production, we would continue the study of charmonium production at the higher energy of the $800 \mathrm{GeV} / \mathrm{c}$ primary proton beam to measure $J / \psi$ and $\chi$ production from a silicon target:

$$
p \mathrm{Si} \rightarrow J / \psi+\text { anything }
$$

and

$$
p \mathrm{Si} \rightarrow \chi+\text { anything }
$$

The measurement of the energy dependence of charmonium production will allow us to more completely verify the production mechanisms of charmonium and the gluon structure function of the nucleon (see Ref. 1). 
E. Finally, using the primary proton beam at the highest energy we plan to search for evidence of hadronic production of $\chi_{b}$ in much the same way that we are measuring charmonium production ${ }^{6,7}$.

\section{Beam Requirements/Experimental Running}

We are basing our estimates of yields on a canonical run of 20 weeks of beam. We anticipate $2.8 \times 10^{8}$ seconds of beam (20 weeks $\times 100$ hours/week $\times 60 \mathrm{~min} / \mathrm{hr} \times 23$ seconds of spill $/ \mathrm{min}$ ). We will use the primary proton beam to take advantage of the maximum energy available at $800 \mathrm{GeV} / \mathrm{c}$. We will require $1.7 \times 10^{8}$ protons per second of spill or $3.8 \times 10^{8}$ protons per spill during data taking. This beam intensity implies a total interaction rate of $2 \times 10^{6}$ interactions per second from our target foils and our silicon tracker. Approximately $1 \times 10^{6}$ interactions per second are generated by each of these devices. We will trigger only on interactions from the silicon target foils by requiring (in the trigger) that there be hits in the first module of the silicon tracker at large angles with respect to the beam. We use the $\mathrm{A}^{0.72}$ dependence of the total cross-section to predict the total cross section in silicon and a total inelastic cross-section for $\mathrm{pN}$ at $800 \mathrm{GeV} / \mathrm{c}$ of $32 \times 10^{-27} \mathrm{~cm}^{2}$ per nucleon.

The interaction rate of $2 \times 10^{6}$ interactions per second to be used in E771 (an interaction every 25 buckets or 470 nanoseconds) is the same as that experienced in our just completed experiment, E705, and is an order of magnitude lower than the interaction rate of experiment E537. Individual parts of the spectrometer such as the electromagnetic calorimeter have been tested in calibration beams at much higher effective rates without degradation. We have achieved a measured suppression due to our fast dimuon trigger and dimuon trigger processor of better than $10^{-4}$ of the raw interaction rate.

We will operate in E771 in much the same mode as we operated E705 with the following exceptions:

1. Our beam tagging Čerenkov counters will not be required during data taking. They will only be used during electron calibration runs for electron identification.

2. We expect to have a much cleaner trigger situation in E771 because of our use of the extracted primary proton beam rather than a pion beam which will decrease substantially the problems due to muon halo.

3. We expect to implement some data filtering at the level of our VME-based microprocessor event builder and to do some online ACP event filtering to reduce the load on the data acquisition system and eliminate many of the background events from being written to tape. 
Finally, as in experiment E705, we will require occasional use of the calibration electron beam for adjustment of our electromagnetic detector. We summarize in Table II below the expected beam usage and the spot sizes that we expect on the experiment target.

Table II: Beam Parameters

\begin{tabular}{|ccccc|}
\hline \hline Proton Beam & Max Flux $/ \mathrm{sec}$ & Flux $/$ spill $(23 \mathrm{sec})$ & $\sigma_{x}(\mathrm{~cm})$ & $\sigma_{y}(\mathrm{~cm})$ \\
\hline $800 \mathrm{GeV} / \mathrm{c}$ & $1.7 \times 10^{8}$ & $3.6 \times 10^{3}$ & 0.3 & 0.2 \\
\hline
\end{tabular}

\section{Experimental Setup}

\section{A. General Remarks}

We plan to use the E705 spectrometer (Figure 1) augmented by a silicon tracker and a multiple silicon foil target (figure 2). We will take advantage of the an upgraded muon trigger system which is being developed for this spectrometer. This trigger, which identifies muon candidates with a transverse momentum above a certain threshold, will provide a suppression of $<10^{-4}$ of the interactions due to the total cross-section. When this trigger is coupled to our data acquisition system which is capable of handling 600 events/sec, we will operate in E771 at an interaction rate of over $2 \times 10^{6}$ interactions per second producing over 300 dimuon and single muon triggers/second from the target. This is a more comfortable manner of operation than that of E705 which requires that we operate at 200 triggers per second in the absence of filtering.

\section{B. Multiple Silicon Foil Target}

We have chosen to use a system of four $3 \mathrm{~mm}$ thick silicon foils as the target system for E771. This system was chosen in order to optimize as much as possible the interaction length of the target while keeping the radiation length of the target below $20 \%$. In addition the higher A of silicon increases the number of bottom events produced relative to a given number of total cross-section interactions. The cross-section for bottom production is assumed to be linear in $\mathrm{A}$ in comparison to the total cross-section which increases ${ }^{21}$ as $\mathrm{A}^{0.72}$. A total of $12 \mathrm{~mm}$ of silicon corresponds to an interaction length of $4.5 \%$ and a radiation length of $20 \%$. Four $3 \mathrm{~mm}$ foils are chosen so that the $B$ decays of interest have will decay outside of the foils in air. Given the long invariant lifetime of $B$ mesons and the Lorentz boost they will have from being produced in a high energy fixed target reaction, a large fraction of these $B$ 's will decay outside of the target foil material. Looking for $J / \psi$ secondary decay vertices in air will eliminate the background due to $J / \psi$ 's produced from secondary interactions in 
the target foil material. The physics signature of a $J / \psi$ vertex found in air outside of any material can only be due to bottom production and decay, with the principal background to this being mis-measured $J / \psi$ vertices.

\section{Silicon Tracker}

Figure 2 shows the configuration of the silicon tracker that will be used in this experiment. This device consists of 24 modules of $300 \mu \mathrm{m}$ thick silicon planes. The size of these three modules will be $1 " \times 1 "$ and $2 " \times 2 "$. The tracker contains approximately $2 \%$ of an interaction length and $8 \%$ of a radiation length. The width of the silicon strips is $25 \mu \mathrm{m}$ in the center and $50 \mu \mathrm{m}$ at the edges. Because of our relatively thin target foils we plan to require in the trigger the presence of wide angle tracks in the gap between foils to insure an interaction in the foils. We will do this by the simple expedient of requiring a certain number of hits in the first silicon module outside of the forward direction.

\section{Event Yield/Backgrounds}

\section{A. $\bar{B} B$ production in $p$ Si interactions at $800 \mathrm{GeV} / \mathrm{c}$}

The number of bottom mesons or hadrons that we will observe is uncertain because of the uncertainty in the hadronic production cross sections for bottom. The calculations of Quigg $^{14}$, Margolis ${ }^{15}$, Combridge ${ }^{16}$, Owens $^{17}$ and Halzen ${ }^{18}$, are shown in Figure 3 along with the current experimental information ${ }^{11,19}$ available on bottom hadroproduction. Table III tabulates the cross sections for 800 and $925 \mathrm{GeV} / \mathrm{c} p p$ interactions predicted for some of these calculations. These cross-sections have been calculated for a quark mass of $5.3 \mathrm{GeV} / \mathrm{c}^{2}$. If the appropriate bottom quark mass to use in these calculations is $4.7 \mathrm{GeV} / c^{2}$, the cross-sections increase by approximately a factor of 2 .

Given the target and beam which are described above, Table IV shows the number of $\bar{B} B$ events that we expect to produce with the canonical $2.8 \times 10^{6}$ seconds of proton beam at $1.7 \times 10^{8}$ protons/sec (375K events per $10^{-33} \mathrm{~cm}^{2}$ of nucleon-nucleon cross-section assuming cross-sections which vary linearly with $\mathrm{A}$ ).

$$
\text { B. } p \mathrm{Si} \rightarrow \bar{B} B+\text { anything: } \bar{B}(B) \rightarrow J / \psi+\text { anything: } J / \psi \rightarrow \mu^{+} \mu^{-}
$$

Using the model for $B$ production outlined in Appendix B, we calculate acceptances of $25 \%$ (and $28 \%$ ) for the two muons resulting from this cascade decay sequence at 800 (and 
Table III: Calculated $B$ Cross-Sections

\begin{tabular}{|llrr|}
\hline \hline Calculation & & $800 \mathrm{GeV} \mathrm{pN}$ & $925 \mathrm{GeV} \mathrm{pN}$ \\
\hline Combridge & $(\Lambda=500 \mathrm{MeV})$ & $2.8 \times 10^{-32}$ & $4.6 \times 10^{-32}$ \\
Owens & $(\Lambda=400 \mathrm{MeV})$ & $2.1 \times 10^{-32}$ & $3.1 \times 10^{-32}$ \\
Owens & $(\Lambda=200 \mathrm{MeV})$ & $9.4 \times 10^{-33}$ & $1.5 \times 10^{-32}$ \\
Margolis & $(\Lambda=200 \mathrm{MeV})$ & $2.7 \times 10^{-33}$ & $4.1 \times 10^{-33}$ \\
Quigg & $(\Lambda=200 \mathrm{MeV})$ & $2.4 \times 10^{-33}$ & $3.8 \times 10^{-33}$ \\
\hline \hline
\end{tabular}

* A K factor of two has been multiplied by the QCD cross sections of Combridge, Owens, and Quigg to take into account higher order processes ${ }^{17}$. Margolis' calculation has been left as is because of its semi-empirical nature.

Table IV: Number of Calculated $\bar{B} B$ events

\begin{tabular}{|llrr|}
\hline \hline Calculation & & $800 \mathrm{GeV} p \mathrm{Si}$ & $925 \mathrm{GeV} p \mathrm{Si}$ \\
\hline Combridge & $(\Lambda=500 \mathrm{MeV})$ & $1.1 \times 10^{7}$ & $1.7 \times 10^{7}$ \\
Owens & $(\Lambda=400 \mathrm{MeV})$ & $8.0 \times 10^{6}$ & $1.2 \times 10^{7}$ \\
Owens & $(\Lambda=200 \mathrm{MeV})$ & $3.5 \times 10^{6}$ & $5.6 \times 10^{6}$ \\
Margolis & $(\Lambda=200 \mathrm{MeV})$ & $1.0 \times 10^{6}$ & $1.5 \times 10^{6}$ \\
Quigg & $(\Lambda=200 \mathrm{MeV})$ & $9.0 \times 10^{5}$ & $1.4 \times 10^{6}$ \\
\hline \hline
\end{tabular}

925) $\mathrm{GeV} / \mathrm{c}$ beam momentum. In calculating the number of $B$ mesons that we will observe decaying into $J / \psi$ 's which subsequently decay into muon pairs, we have used $1.10 \%$ (the average of the two existing measurements) for the branching ratio for $B$ and $7 \%$ for the branching ratio for the $J / \psi$ decay mode. In addition, we have taken into account the factor of 2 that comes from having both a $B$ and a $\ddot{B}$ in the final state. Note that we have also assumed that the inclusive branching ratio for $B^{ \pm}, B^{0}$, and $B_{s}^{0}$ into $J / \psi+\mathrm{X}$ are the same. Under this assumption and the assumption that these three types of $B$ mesons dominate the hadronization of bottom quarks, we can calculate the number of $B$ meson decays that we expect to see in our canonical run. We tabulate these numbers in Table V below.

Table V: Number of $B \rightarrow J / \psi+\mathrm{X} ; J / \psi \rightarrow \mu^{+} \mu^{-}$

\begin{tabular}{|llrr|}
\hline \hline Calculation & & $800 \mathrm{GeV} p$ Si & $925 \mathrm{GeV} p \mathrm{Si}$ \\
\hline Combridge & $(\Lambda=500 \mathrm{MeV})$ & 4240 & 7330 \\
Owens & $(\Lambda=400 \mathrm{MeV})$ & 3080 & 5170 \\
Owens & $(\Lambda=200 \mathrm{MeV})$ & 1350 & 2410 \\
Margolis & $(\Lambda=200 \mathrm{MeV})$ & 390 & 650 \\
Quigg & $(\Lambda=200 \mathrm{MeV})$ & 350 & 600 \\
\hline
\end{tabular}


All of these numbers must be multiplied by an efficiency for surviving the vertex cuts which are necessary to isolate true bottom decays with a clean, unconfused secondary vertex in the silicon tracker and to eliminate false secondary vertices from "ordinary" $\mathrm{J} / \psi$ decays. In addition, all the other usual standard reconstruction efficiencies such as those due to PWC's, drift chambers, etc. must be taken into account. We now discuss the identification and reconstruction of the secondary vertices in the $B \rightarrow J / \psi \rightarrow \mu^{+} \mu^{-}$decays.

We have estimated the resolutions with which a decay vertex can be reconstructed and the efficiency with which it can be separated from the silicon foils and the other vertices in the event by the use of a Monte Carlo incorporating the $B$ production and decay model of Appendix B (which uses a $0.6 \times 10^{-12}$ second for the bottom lifetime). This Monte Carlo incorporates the expected resolution of the silicon tracker and the multiple scattering in the foil target and the silicon tracker. The resolution for reconstruction of secondary vertices using only the muon tracks from the $J / \psi$ is calculated to be:

$$
\begin{aligned}
& \sigma_{x, y} \approx 17 \mu \mathrm{m} \\
& \sigma_{r} \approx 13 \mu \mathrm{m} \\
& \sigma_{z} \approx 480 \mu \mathrm{m}
\end{aligned}
$$

Here $x$ and $y$ are the transverse dimensions, $r=\sqrt{x^{2}+y^{2}}$ and $z$ is the dimension in the beam direction. The distributions of the residuals relative to the true position of the vertex of the decay in these coordinates are given in Figures 4a, b, c, and d. To give a sense of the distances involved in $B$ decay, we show in Figure $4 \mathrm{a}$ and $\mathrm{b}$ the $\Delta z$ and $\Delta r$ separations of the true decay vertices from the true primary vertex.

In the same way we have calculated the average residuals for primary vertices. Here as a model for $800 \mathrm{GeV} / \mathrm{c} p N$ interactions we have used $360 \mathrm{GeV} / \mathrm{c}$ bubble chamber events scaled up to $800 \mathrm{GeV} / \mathrm{c}$. (The multiplicities are scaled by $\log s$ and the momentum of the secondaries by $\sqrt{s}$ ). We observe the $\Delta x, \Delta y$, and $\Delta z$ distributions shown in Figures $6 \mathrm{a}, \mathrm{b}$, $c$, and $\mathrm{d}$. The widths of these distributions are:

$$
\begin{array}{ll}
\sigma_{x, y} & \approx 7 \mu \mathrm{m} \\
\sigma_{r} & \approx 5 \mu \mathrm{m} \\
\sigma_{z} & \approx 200 \mu \mathrm{m}
\end{array}
$$

The residual distributions for the primary vertex are narrower than those of the secondary vertices because of the larger number of outgoing tracks.

Given these resolutions and the scale of the events, reasonable efficiencies for recognition of events with secondary vertices due to bottom decay can be achieved. A scatterplot of the $\Delta z$ and $\Delta r$ separation between the reconstructed primary and secondary decay vertices for typical bottom events (using only the decay muons for the secondary vertex) is shown in Figure 7a. The projections of this scatterplot are shown in Figures $7 \mathrm{~b}$ and $7 \mathrm{c}$. These projections can be compared to the true separations plotted in Figures $5 \mathrm{a}$ and $\mathrm{b}$. In the Monte Carlo calculation used to obtain these distributions we have superimposed bottom decays of the type $B \rightarrow J / \psi K \pi$ on the scaled bubble chamber events in order to get a realistic 
estimate of the reconstruction difficulties in these complicated events. In this Monte Carlo, smearing effects due to the misassignment of tracks from the other $B$ decay to the primary vertex are included. A cut of $\Delta z=1 \mathrm{~mm}$ and $\Delta r=50 \mu \mathrm{m}$ will retain $76 \%$ of the true bottom decays. Making a conservative estimate of composite efficiency (geometric acceptance is already taken into account) of the rest of the spectrometer (PWC's, drift chambers, etc.) of $80 \%$, we expect to collect $\approx 60 \%$ of the $B \rightarrow J / \psi \rightarrow \mu^{+} \mu^{-}$decays given in Table $\mathrm{V}$.

In order to estimate the backgrounds to this signal, we have superimposed "ordinary" $J / \psi$ production on the $800 \mathrm{GeV} / \mathrm{c}$ events and estimated the number of $J / \psi$ 's that will appear to form a false secondary vertex. Figures $8 \mathrm{a}, \mathrm{b}$, and $\mathrm{c}$ give the scatterplot and projections for reconstructed $J / \psi$ 's from the primary vertex corresponding to the scatterplot and projections for the true decays (figures $7 \mathrm{a}, \mathrm{b}, \mathrm{c}$ ). The cuts of $\Delta r>50 \mu \mathrm{m}$ and $\Delta z>1 \mathrm{~mm}$ which were chosen to select events containing true secondary vertices, reject $2.8 \times 10^{-6}$ of all "ordinary" $J / \psi$ events. In addition, if necessary, we can require that there be a third track that appears to come within $50 \mu \mathrm{m}$ of the secondary vertex defined by the muons. This additional criterion reduces the remaining background from poorly reconstructed $J / \psi$ 's by an additional factor $>10$. Only a slight loss of signal is caused by this third track requirement. Thus we have an overall suppression of background $2.8 \times 10^{-6}$.

Another experimental issue is the efficiency with which the muons, as identified and measured in the spectrometer proper, can be identified with a particular track as measured in the tracker. We have investigated the difficulties in matching the silicon detector tracks and the tracks as detected in the PWC's and drift chambers of the spectrometer. We have attempted to make matches both in angle and in intercept at various planes downstream of the last module of the silicon tracker. We conclude that there is little confusion in correlating the muon tracks with the appropriate tracks in the silicon tracker. We estimate $<5 \%$ loss of dimuon events due to inability to correlate the muons with the correct tracker track.

In conclusion, after these cuts and efficiencies take their toll, we are left with a few hundred to a few thousand inclusive $B \rightarrow J / \psi \rightarrow \mu^{+} \mu^{-}$decays (depending on the exact level of the cross-section) with which to estimate cross-sections for hadroproduction of bottom mesons. If the majority of these $B^{\prime}$ 's are bottom mesons $B^{ \pm}, B^{0}$, or $B_{s}^{0}$, then there are several exclusive decay modes (see Table I) which have relatively simple topologies and which take place at the level of $10 \%$ of the $B \rightarrow$ anything sample of events. It seems quite feasible to reconstruct the $B$ mesons which decay into $x^{+} y^{-}$or $J / \psi x^{+} y^{-}$. Some smaller portion of the events which have a single $K^{0}$ in the final state may be reconstructable. These $K^{0}$ events have the advantage that there is no two-fold ambiguity in the identity of the strange particle which plagues the totally charged final states in the absence $K-\pi$ identification. Those final states having more than one $K^{0}$ or $\pi^{0}$ are much more difficult to reconstruct. We conclude that obtaining samples of several exclusive bottom decay modes containing a few 10's to a few 100's of totally reconstructed events seems definitely within the realm of feasibility.

Using the suppression of background obtained from the secondary vertex cuts and the requirement of a third track pointing at the secondary vertex, we estimate, using the number 
of $J / \psi$ 's from Table $V$ that are produced in our standard run, that we will have a background of a few 10's of fake events to our inclusive signal of a few hundred to a few thousand true $B \rightarrow J / \psi \rightarrow \mu^{+} \mu^{-}$events. The background will be further reduced in our search for specific masses. Our mass resolution should be quite good, on the order of $20-30 \mathrm{MeV} / \mathrm{c}^{2}$ for a final state such as $B^{-} \rightarrow J / \psi K^{-}$, because the technique of fixing the $J / \psi$ mass after the $J / \psi$ is observed can be employed. This improvement in mass resolution should make negligible any remaining backgrounds to the exclusive decay signals.

\section{C. $p \mathrm{Si} \rightarrow \bar{B} B+\mathrm{X}: \bar{B}^{0} B^{0}$ Mixing as Detected by the Semileptonic Decay $B^{0} \rightarrow \mu+\nu+\mathrm{X}$ Leading to Same Sign Dimuons}

The signal for this physics is same sign dimuons where each muon comes from a different neutral secondary vertex. The $B^{0}$ or $\bar{B}^{0}$ evolve into one another and then decay semileptonically thereby producing a same sign dimuon signal.

The semileptonic branching ratio for the $B$ mesons is $12.4 \%$. We assume that this branching ratio is approximately the same for $B_{s}$. If we look for the simultaneous semileptonic decay of both the $B$ 's to detect $B^{0} \bar{B}^{0}$ mixing, we will observe $1.54 \times 10^{-2}$ of all produced $\bar{B} B$ final states undergoing double semileptonic decay and producing opposite sign dimuons. If some of the $B^{0}$ 's evolve into their antiparticles then we will observe a same sign dimuon signal which is some fraction of this opposite sign dimuon signal. It is now known ${ }^{20,21}$ that there is strong mixing between $\bar{B}^{0}$ 's and $B^{0}$ 's. The level of the mixing of the strange bottom mesons $\left(B_{s}^{0}\right.$ and $\left.\bar{B}_{s}^{0}\right)$ is expected to be maximal ${ }^{20,21}$. If $B^{0}, B^{ \pm}$, and $B_{s}^{0}$ are produced equally copiously in the hadronization of the $b$ quarks in $p N$ interactions, then $\frac{4}{9}$ of the possible $\bar{B} B$ final states will include one $B_{s}^{0}$ meson that can evolve into a $\bar{B}_{s}^{0}$ meson and $\frac{1}{9}$ of the final $\bar{B} B$ states will have two $B_{s}^{0}$ mesons. Therefore, we would expect to see a like sign dimuon signal due to $B^{0} \bar{B}^{0}$ mixing which is $1.89 \times 10^{-3}$ of all $\bar{B} B$ final states. The geometric acceptance for muons from double semileptonic decays of bottom has been estimated to be $25 \%$. So we expect to see between 425 and 5200 same sign dimuons from mixing in comparison to the 3500 to 42,400 opposite sign dimuons from the double semileptonic decays of $B$ 's produced in $800 \mathrm{GeV} / \mathrm{c}$ interactions depending on the cross-section for bottom. For $925 \mathrm{GeV} / \mathrm{c}$ $p$-Si interactions we would expect to observe between 660 and 8100 same sign dimuons from mixing in comparison to 8200 to 40,500 opposite sign dimuons.

The backgrounds to $\bar{B} B$ mixing come from several sources. The most serious background is produced by the sequential decay of one of the $B$ 's to a charmed daughter which decays semileptonicaly into a muon which has a different sign than would have been produced by the semileptonic decay of the parent $B$. This will produce a same sign dimuon background if the other $B$ also decays semileptonically. There are several decay chains which result in this sort of "daughter charmed particle" same sign background to $\bar{B} B$ mixing. Under the assumptions 1 ) that $B^{ \pm}, B^{0}$, and $B_{s}^{0}$ are made equally frequently; and 2) that the various reported branching ratios (the ratios that are used are either preliminary results from the 
CLEO experiment ${ }^{22}$ or reported ratios from the particle data tables ${ }^{23}$ ) for decays of unseparated mixtures of $B^{0}, \bar{B}^{0}, B^{+}, B^{-}$can be used both for the individual bottom mesons and for $B_{s}^{0}$ mesons; we can evaluate the backgrounds due to the decay sequences listed below:

- Charged $B$ plus neutral $B$ production followed by decay of $B^{+} \rightarrow \bar{D}^{0}$. $2.6 \times 10^{-3}$ of the $B^{+} \bar{B}^{0}$ production ( $\frac{4}{9}$ of all $b \bar{b}$ pairs) decays via these chains to produce a background of 260 to 3200 same sign dimuons in a canonical $800 \mathrm{GeV} / \mathrm{c}$ run.

- Charged $B$ plus neutral $B$ production followed by decay of $B \rightarrow D^{ \pm}$. An additional $3.5 \times 10^{-3}$ of the $B^{+} B^{0}$ production ( $\frac{4}{9}$ of all $b \bar{b}$ production) will decay via charged $D$ 's into a same sign dimuon background of 350 to 4300 events in the $800 \mathrm{GeV} / \mathrm{c}$ run.

- Charged $B$ plus neutral $B$ production followed by decay of $B^{0}, \bar{B}^{0} \rightarrow \bar{D}^{0}, D^{0}$. This decay chain is responsible for an additional $2.6 \times 10^{-3}$ of the $B^{+} \bar{B}^{0}+B^{-} B^{+}$ production decaying to a same sign background of 260 to 3200 events.

- Charged $B$ plus neutral $B$ production followed by decay of $B^{0}, \bar{B}^{0} \rightarrow D^{ \pm}$. This decay chain is responsible for an additional $3.5 \times 10^{-3}$ of the $B^{0} \bar{B}^{0}$ production decaying to a background of 350 to 4300 events.

- Neutral $B$ production followed by decay of $B^{0}, \bar{B}^{0} \rightarrow \bar{D}^{0}, D^{0}$. This decay sequence results in $2.6 \%$ of the $B^{0} \bar{B}^{0}$ final states $\left(\frac{4}{9}\right.$ of the total $b \bar{b}$ production) decaying into a same sign background which contains 260 to 3200 events for the $800 \mathrm{GeV} / \mathrm{c}$ run.

- Neutral $B$ production followed by decay of $B^{0}, \bar{B}^{0} \rightarrow D^{-}, D^{+}$. $3.5 \times 10^{-3}$ of the $B^{0} \bar{B}^{0}$ production ( $\frac{4}{9}$ of the entire $b \bar{b}$ production) decays via this chain to a same sign dimuon background to 350 to 4300 events.

- Charged $B$ production followed by decay of $B^{ \pm} \rightarrow \bar{D}^{0}, D^{0}$. $3.5 \times 10^{-3}$ of the $B^{+} B^{-}$final states ( $\frac{1}{9}$ of all final $b \bar{b}$ states) decay via this sequence to a same sign dimuon background of 90 to 1070 events.

- Charged $B$ production followed by decay of $B^{ \pm} \rightarrow D^{-}, D^{+}$. $2.6 \times 10^{3}$ of the $B^{ \pm}$final states ( $\frac{1}{9}$ of all $b \bar{b}$ final states) decay via this sequence producing a same sign dimuon background of 70 to 800 events.

Thus, the total same sign background from the sum of these sequences, in the absence of any cuts to suppress them, would be 2000 to 24000 events to be compared to a $B^{0} \bar{B}^{0}$ mixing signal of 660 to 8100 events in an $800 \mathrm{GeV} / \mathrm{c}$ run. So our signal to background is $\frac{1}{3}$ from the charmed daughter decay sequences without cuts.

The second same sign background to $\bar{B} B$ mixing is production of $D^{0} \bar{D}^{0}$ followed by mixing and the subsequent semileptonic decay of the charmed particles. In this case the semileptonic decay branching ratio is $5.3 \%$ for the $D^{0}$ and the mixing between $D^{0}$ and $\bar{D}^{0}$ 
is small $\left(<0.006\right.$ experimentally ${ }^{24}$ and estimated theoretically ${ }^{20,21}$ to be between $10^{-4}$ and $10^{-7}$ ). Taking the largest theoretical rate and assuming that we have the same acceptance (25\%) for the background dimuons as for the mixing dimuons, we would expect to see a same sign background of 60 to 770 like sign dimuons from $D^{0} \bar{D}^{0}$ mixing even if the charm cross-section is 1000 times as large as the bottom cross-section at $800 \mathrm{GeV} / \mathrm{c}$.

The third background of $\bar{B} B$ mixing that we have considered is produced when one of the $B$ 's decays semileptonically and the other $B$ decays nonleptonically. Then if one of the pion decay products of the second $B$ decay itself decays into $\mu \nu$, a same sign background can be produced. Approximately $1 \%$ of the bottom decays produces a muon via $\pi \rightarrow \mu \nu$ so that we would expect 30 to 340 same sign background events from this source.

We have examined the possibility that we can further suppress these backgrounds (especially the "daughter" backgrounds) by cutting to the high mass muon pairs which should be relatively rich in the muons from bottom decay. Using the $\bar{B} B$ production model of appendix $\mathrm{B}$, we have generated the like sign dimuons mass spectrum for the $\bar{B} B$ mixing signal and for each of the three backgrounds mentioned above: $B \rightarrow C \rightarrow \mu$ mixing and $B \rightarrow \pi \mu$. These four dimuon mass spectra are shown in Figure 9. These spectra have arbitrary normalizations with respect to each other but the shapes of the spectra can be compared. For masses above $3 \mathrm{GeV} / \mathrm{c}^{2}, 42 \%$ of the true mixing signal is retained while only $19 \%, 19 \%$, and $25 \%$, respectively, of the three backgrounds survive. Therefore, improvements of a factor of two are realized in the ratio of signal to background in the high mass region.

A second technique for suppression of these backgrounds to $\bar{B} B$ mixing is the reconstruction of the "visible" mass of the secondary vertex. We show in Figures 10a and b the visible mass of the secondary vertex from

$$
B^{0} \rightarrow K \pi \pi \mu \nu
$$

in comparison to the visible mass from

$$
D^{0} \rightarrow K \pi \mu \nu
$$

if the correct assignment of the right tracks to a given secondary vertex can be accomplished. These figures show that for a perfect assignment of tracks, $98 \%$ of the bottom secondary decay vertices with this topology have masses above $2.0 \mathrm{GeV} / \mathrm{c}^{2}$ and can be cleanly separted from the $D^{0}$ decays with the same topology. In addition, if a $100 \%$ correct assignment of tracks to specific vertices could be accomplished, then one could require the neutrality of each pair of secondary vertices with a muon track before the event was added to the mixing sample of events. This would eliminate all but the daughter decay sequences 5 and 6 exclusive of the irreducible backgrounds due to charged $D$ 's decaying too near to their parent $B^{0}$ decays to be resolved or of neutral $D$ 's decaying too far from the parent $B^{ \pm}$decay to be associated with that parent. Figures $11 \mathrm{a}$ and $\mathrm{b}$ show the longitudinal and transverse separation of the daughter $D$ decay vertices from their parent vertices.

However, a completely correct assignment of all tracks associated with a particular secondary vertex to that vertex is very difficult since mistakes can be made either by adding 
tracks or losing them. This is especially true for the mixing event topology ${ }^{3}$ in distinction to the $J / \psi$ topology in which there are only parts of muons from different vertices to flag the events and to begin the process of reconstruction of a secondary vertex. If one can begin by developing a technique for finding secondary vertices in such events then the association of the tracks with these vertices can proceed given the track resolutions possible with silicon trackers. We show in Figure $12 \mathrm{a}, \mathrm{b}, \mathrm{c}$, and $\mathrm{d}$ the distance from the true decay vertex that the reconstructed primary and secondary tracks pass. As a first attempt at solving this problem, we have partially investigated one possible technique for defining secondary vertices and assigning tracks to them. The steps in this iterative process are as follows:

1. Form a first order primary vertex from fits of all tracks in the event excluding the muon tracks. The resolution of the primary vertex for $\bar{B} B$ events is $\approx 21 \mu \mathrm{m}$ due to the broadening by the tracks from the secondary vertices.

2. Choose tracks to be associated with the primary vertex by excluding those at distances greater than $50 \mu \mathrm{m}$ from the first order vertex.

3. Form the second order primary vertex by fitting this track sample. The second order vertex resolution now is $7.3 \mu \mathrm{m}$ in agreeement with the true resolution for primary vertices.

4. Demand that the muon track be separated by $50 \mu \mathrm{m}$ in the transverse dimension at the target midplane from this second order vertex just formed. As shown in Figure $13 \mathrm{a}$ and $\mathrm{b}$, this cut loses $50 \%$ of the muons from $B$ decay but eliminates $98 \%$ of the tracks from the primary vertex from candidacy for secondary tracks.

5. Demand that any track that is to be asociated with the secondary muon pass within a distance $\sqrt{\left(\Delta x^{2}+\Delta y^{2}+\Delta z^{2}\right)}=50 \mu \mathrm{m}$ of the muon at the distance of closest approach.

Using these criteria we have selected decay muons and associate secondary tracks with those muons for samples of events containing $\bar{B} B$ decays and $D \bar{D}$ decays into $K \pi \pi \pi \mu \nu$ to test the technique. For the sample of chosen tracks which appear to be associated with the decay muons we have formed the visible mass. Figures $15 \mathrm{a}$ and $\mathrm{b}$ show this mass for these samples. We see that the " $B$ " masses go below $2 \mathrm{GeV} / \mathrm{c}^{2}$ because of the loss of tracks, and far above $5.3 \mathrm{GeV} / \mathrm{c}^{2}$ because of the inclusion of tracks not belonging to the secondary vertex. Obviously, more iterations would help to eliminate these losses and inclusions as the fits of the vertex improve. However, even at this relative simple stage with relatively naive algorithm for determining a vertex, the visible mass is still a useful tool. A cut at $3 \mathrm{GeV} / \mathrm{c}^{2}$ will eliminate $40 \%$ of the $D$ mesons while keeping $88 \%$ of the $B$ decays.

Therefore, use of this crude version of visible mass should make another factor of 2 in mixing signal to background ratio. Taken in conjunction with the selection of high mass events, we should achieve signal to background ratios greater than 1 for limited samples $(\approx$ 
$10 \%$ ) of the same sign events. It should, however, be possible to find better procedures and algorithms to define the secondary vertices and associate the proper tracks with them.

\section{D. $p \mathrm{Si} \rightarrow \chi+\mathrm{X}: \chi \rightarrow J / \psi \gamma: J / \psi \rightarrow \mu^{+} \mu^{-}$}

The study of the production of $\chi$ states that we are just finished data taking on will be continued at higher energies with the extracted primary proton beam. The measurement of hadroproduction of $\chi$ 's allows us to study gluon fusion and the structure function of the gluon, especially in the case of $p N$ interactions. We refer the reader to the E705 proposal ${ }^{1}$ for a more extensive discussion of this topic. We have data with protons at $300 \mathrm{GeV} / \mathrm{c}$, so energy dependent effects can be studied. We list in Table VI our expectations for data at 800 and $925 \mathrm{GeV} / \mathrm{c}$ assuming that $30.5 \%$ of the observed $J / \psi$ 's are produced from the decays of $\chi$ 's via the $J / \psi \gamma$ decay mode just as is the case at lower energies. The A dependence used to scale these cross sections to the silicon target is $\mathrm{A}^{0.32}$.

Table VI: $\chi$ Cross-Sections and Acceptances

\begin{tabular}{|rrrrr|}
\hline Energy & $\sigma(p N \rightarrow J / \psi)$ & $\chi$ Acceptance & $N_{J / \psi \rightarrow \mu \mu}$ & $N_{\chi \rightarrow J / \psi \gamma \rightarrow \gamma \mu \mu}$ \\
\hline $800 \mathrm{GeV} / \mathrm{c}$ & $600 \mathrm{nb}$ & 0.132 & $\approx 1.4 \times 10^{6}$ & $4.2 \times 10^{5}$ \\
$925 \mathrm{GeV} / \mathrm{c}$ & $800 \mathrm{nb}$ & 0.110 & $\approx 1.5 \times 10^{6}$ & $4.6 \times 10^{5}$ \\
\hline \hline
\end{tabular}

E. $p \mathrm{Si} \rightarrow \chi_{b}+\mathrm{X}: \chi_{b} \rightarrow \Upsilon \gamma: \Upsilon \rightarrow \mu^{+} \mu^{-}$

The same sequences of decays that were used to detect $\chi$ can be used to detect $\chi_{b}$ states of the bound $\bar{b} b$ system. Using the model for $\Upsilon(9460)$ production in $p p$ interactions of reference 15 which has been fitted to the experimental data of reference 25 and 26 , we predict $\sigma(p p \rightarrow \Upsilon+\mathrm{X}) \approx 6 \times 10^{-34} \mathrm{~cm}^{2}$ at $800 \mathrm{GeV} / \mathrm{c}$ and $8 \times 10^{-34} \mathrm{~cm}^{2}$ at $925 \mathrm{GeV} / \mathrm{c}$. These cross-sections yield approximately 22,500 and $30,000 \Upsilon$ 's at 800 and $925 \mathrm{GeV} / \mathrm{c}$ for our canonical run. The branching ratio ${ }^{23}$ is $\mathrm{BR}\left(\Upsilon \rightarrow \mu^{+} \mu^{-}\right)=0.0291$, so the total number of $\Upsilon \rightarrow \mu^{+} \mu^{-}$observed in our run will be 650 or 900 at 800 or $925 \mathrm{GeV} / \mathrm{c}$ respectively. While the number of $\chi_{b}$ which cascade through the decay chain $\chi_{b} \rightarrow \Upsilon \gamma \rightarrow \gamma \mu \mu$ is unknown, an inspection of the $\chi_{b}$ branching ratios into $\Upsilon_{\gamma}$ makes it tempting to surmise that $\frac{1}{3}$ of the $\Upsilon$ production occurs through this sequence (as is the case for $\chi \rightarrow J / \psi \gamma$ ). If this is the case we can expect 200 or $300 \chi_{b} \rightarrow \Upsilon_{\gamma} \rightarrow \gamma \mu \mu$ before acceptance cuts and reconstruction efficiencies are imposed. Making reasonable estimates for these factors, we should be left with several 10's of events at these energies. This would be sufficient for a first observation of the hadroproduction of $\chi_{b}$. 


\section{Conclusions}

The study of the heavy quark states produced using the highest energy proton beam available with the E705 spectrometer augmented with silicon offers an exciting opportunity. This spectrometer is particularly well suited because of its long history of studying dimuon final states to look for bottom hadrons associated with dimuons. The addition of a silicon tracker is necessary in order to detect the secondary vertices and the presence of a $J / \psi$ produced in such an isolated secondary vertex will ensure that a bottom decay is being observed. The sophistication of our dimuon and single muon trigger system and the development of a data acquisition system capable of handling large data flows make this experiment uniquely able to run at a rate (in an open geometry) necessary to make it accessible to bottom cross-sections. 


\section{References}

1. M. Binkley et al., Fermilab Proposal E-705, (Oct. 1981)

2. H. Areti et al., "A Fast Trigger Processor for Dilepton Triggers," Nucl. Instr. Meth., A212, 135 (1983).

3. E. Anassontzis, et al., "A Large Aperture Spectrometer to Study Dimuons," Nucl. Instr. Meth., A242, 215 (1985).

4. E. Anassontzis, et al., "Continuum Dimuon Production in $\bar{p} W$ Collisions at $125 \mathrm{GeV} / \mathrm{c}$, Phys. Rev. Lett., 54, 2572 (1985).

5. P. Karchin, "Performance of a Silicon Microstrip Vertex Detector in a Charm Photoproduction Experiment: Fermilab E691," to be published in the Proceedings of the UCLA Workshop on SSC Physics, (Jan. 1986).

6. B. Cox, "The High Intensity Laboratory," Fermilab Report 79/1, 0091.1, (1979).

7. ARGUS Collaboration, DESY 85-070, also see R. Davis, Proceedings of the Amer. Phys. Soc., Eugene, Oregon (Aug. 1985) to be published. Also see R. Orr, Proceedings of the Lake Louise Winter Institute, Alberta, Canada (1986).

8. CLEO Collaboration, also see R. Davis, Proceedings of the Amer. Phys. Soc., Eugene, Oregon (Aug. 1985) to be published. Also see M.S. Alam, Proceedings of the Lake Louise Winter Institute, Albert, Canada (1986).

9. J. P. Albanese, et al., WA75 Collaboration, CERN/EP 85-76.

10. E. H. Thorndike, to be published in the Proceedings of the 1985 International Symposium on Leptons and Photon Interactions, Kyoto (Aug. 1985).

11. C. Caso, "New Flavor Production in Hadronic Interactions," CERN/EP 180, (Nov. 1985).

12. J. D. Bjorken, private communication, (July 1985).

13. M. G. D. Gilchriese, private communication, CBX 86-17.

14. C. Quigg, Memo to Taiji Yamanouchi, (July 16, 1985).

15. Y. Afek, C. Leroy and B. Margolis, Phys. Rev. D, Vol. 22, 1, 86 (1982).

16. B. L. Combridge, Nucl. Phys. B151, 429, (1979).

17. J. Owens, private communication.

18. F. Halzen, "Production of Heavy Quarks," Proceedings of the 21st International Conference on High Energy Physics, Paris, CS-381 (1982). 
19. D. Treille, "Hard Scattering of Hadrons and Photons," Proceedings of the International Conference on High Energy Physics, Bari, Sicily (1985).

20. A. Ali and C. Jarlskog, Phys. Lett., 144B, 266, (1984).

21. A. Ali, "Heavy Flavor Production and Weak Mixings," Proceedings of the International Symposium on the Physics of $\bar{p} p$ Collisions, Tsukuba, 456, (March 1985).

22. M. G. D. Gilchriese, private communication.

23. C. G. Wohl et al., "Reviews of Particle Properties," Reviews of Modern Physics, 56, S190 (1984).

24. W. C. Louis et al., Phys. Rev. Lett., 56, 1027 (1986).

25. K. Ueno et al., Phys. Rev. Lett., 42, 486 (1979).

26. D. Antreasyan et al., CERN Report, EP/79 116 (1979).

27. T. Toohig, "Fermilab Magnets, Power Supplies, and Auxilliary Devices: Technical Data," Fermilab TM-632 (1975).

28. D. C. Carey, TURTLE (Trace Unlimited Rays Through Lumped Elements), Fermilab TM-64, (1971). 


\section{Prairie View High Energy Physics Publications from 1989-1992}

The following publications were produced by Prairie View A\&M University High Energy Physics collaborators during 1989-1992 with Department of Energy support.

- L. Antoniazzi, et al., "Measurement of $J / \psi$ and $\psi^{\prime}$ production in $300-\mathrm{GeV} / \mathrm{c}$ proton, antiproton, and $\pi^{ \pm}$interactions with nuclei”, Phys. Rev. D, 46, 4828 (1992).

- T. Alexopoulos, et al., “B Physics at FNAL E771”, Nucl. Phys. B27, 257 (1992).

- L. Antoniazzi, et al., "The Experiment 705 Spectrometer: A Large Aperture Spectrometer at Fermilab to Study High Mass Dimuons and High Transverse Momentum Photons", Nucl. Instrum. Meth. A315, 92 (1992).

- D. Wagoner, "Design of a Muon Detector for a Fixed Target $B$ Experiment", Proceedings of the $B$ Physics at Proton Accelerators Workshop I: Experiments in a $20 \mathrm{TeV}$ Extracted Proton Beam, DeSoto, 187 (1992).

- L. R. Fortney, et al., "High $p_{T}$ Production of $\pi^{0}$ and $\eta$ at $300 \mathrm{GeV} / c$ ", Proceedings of the 1991 Meeting of the Division of Particles and Fields of the American Physical Society, Vancouver, 669 (1991).

- M. Rosati, et al., "Production of Charmonium States from Experiment 705 at Fermilab", Proceedings of the 1991 Meeting of the Division of Particles and Fields of the American Physical Society, Vancouver, 672 (1991).

- L. Spiegel, et al., "A Combination Drift Chamber/Pad Chamber for Very High Readout Rates", FERMILAB-TM-1765, (1991).

- Q. Shen, et al., "High Transverse Momentum Pizero and Direct Photon Production", Proceedings of the XXI International Symposium on Multiparticle Dynamics, Wuhan, PRC, (1991).

- T. Murphy, et al., "Preliminary Results from Fermilab E-705", Proceedings of the XXVIth Rencontre de Moriond, Les Arcs, France, (1991).

- G. Zioulas, et al., "High Transverse Momentum Pizero and Direct Photon Productions from E705", Proceedings of the Direct Photon Workshop, Fermilab, (1991).

- P. Lebrun, et al., "Bottom Collider Detector at the SSC: An intermediate and low $P_{t}$ detector designed for $B$ physics", Proceedings of the 1990 Meeting of the Division of Particles and Fields of the American Physical Society, Houston, 1065 (1990).

- J. N. Butler, et al., "Report of the Fixed Target Heavy Quark Working Group", Physics at Fermilab in the 1990's, Breckenridge, 418 (1990). 
- H. Castro, et al., "Expression of Interest for A Bottom Collider Detector at the SSC", SSC EOI-11, (1990).

- H. Castro, et al., "Bottom Collider Detector (BCD)-An Intermediate- and Low- $P^{t}$ Detector for the SSC", SSC-240, (1989).

- H. Castro, et al., "The Bottom Collider Detector (BCD)", Fermilab Technical Memo TM-1628, (1989).

- D. Wagoner, "Considerations for a Muon System for BCD at the SSC", BCD Note and Proceedings of the Workshop on $B$ Physics in $p-p$ Collisions at the SSC, DeSoto, 297 (1989).

- D. Wagoner, "Particle Distributions for $20 \mathrm{TeV}$ Fixed Target Interactions", Workshop on a $20 \mathrm{TeV}$ Fixed Target $B$ Physics Facility for the SSC, Fermilab, (1989).

- L. Spiegel, et al., "Performance of a Lead Radiator, Gas Tube Calorimeter", IEEE Trans. Nucl. Sci. NS-36, 86 (1989).

- S. Conetti, et al., "Performance of $0.75 \mathrm{~mm}$ Pitch MWPC's Operating at High Rate", IEEE Trans. Nucl. Sci. NS-36, 112 (1989).

- C. M. Jenkins, et al., "Results from the E-705 Electromagnetic Shower Position Detector", IEEE Trans. Nucl. Sci. NS-36, 117 (1989).

- G. Zioulas, et al., "An On-Line Trigger Processor for Large Transverse Energy Events", IEEE Trans. Nucl. Sci. NS-36, 375 (1989).

- S. W. Delchamps, et al., "Precision Charge Amplification and Digitization System for a Scintillating and Lead Glass Array", IEEE Trans. Nucl. Sci. NS-36,-680 (1989).

- R. L. Wagner, et al., "Nuclear-Target Effects in $J / \psi$ Production in $\bar{p}$ and $\pi^{-}$Interactions", Nucl. Phys. A498, 261c (1989).

- S. Conetti, et al., "Charmonium and Direct Photon Production from Fermilab E-705", Proceedings of the XXIVth Rencontre de Moriond, Les Arcs, France, (1989).

- L. Spiegel, et al., "Charmonium Hadroproduction", Physics in Collision 9, Jerusalem, (1989).

- T. Pramantiotis, et al., "Hadronic Producton of Charmonium at $300 \mathrm{GeV} / c$ ", Bull. Amer. Phys. Soc. 34.4, 1208 (1989).

- G. Zioulas, et al., "Direct Photon Production in $\pi{ }^{7} \mathrm{Li}$ Interactions at $300 \mathrm{GeV} / c$ ", Bull. Amer. Phys. Soc. 34.4, 1208 (1989). 